\title{
COMPLICATIONS ORBITAIRES DE LA POLYPOSE NASOSINUSIENNE DE L'ENFANT
}

\author{
J. MOALLA, H. DHOUIB, S. KALLEL, M. SELLAMI, I. CHARFEDDINE, A. GHORBEL
}

SERVICE ORL, CHU HABIB BOURGUIBA. SFAX. TUNISIE.

\begin{abstract}
La polypose nasosinusienne (PNS) est une pathologie rare chez l'enfant. La symptomatologie clinique est habituellement celle de l'adulte. Exceptionnellement, une complication locorégionale lui est révélatrice. A travers deux observations de PNS diagnostiquées suite à une complication orbitaire, on discute les particularités cliniques de cette entité ainsi que ses implications thérapeutiques.

II s'agissait de deux enfants âgés de 13 ans ayant été hospitalisés pour une exophtalmie unilatérale d'apparition aiguë. L'examen clinique a découvert une PNS surinfectée évoquant alors le diagnostic de complication orbitaire d'origine infectieuse, confirmé par l'imagerie. Le traitement a associé un drainage chirurgical d'urgence de l'abcès orbitaire par voie externe paracanthale interne, suivi par une corticothérapie locale. L'ethmoïdectomie a été nécessaire dans un cas. L'évolution était favorable dans les deux cas avec une stabilisation de la PNS et une absence de signes de récidive de l'infection orbitaire.
\end{abstract}

Sinusonasal polyposis ( PNS) is rare in children. Clinical signs is same that in adults. Exceptionally, it is revealed by a regional complication. Through two cases of PNS with orbital complication, we discuss the clinical and therapeutic particularities of such situation.

It was about two Childs of 13 years, accepted in hospital for an acute unilateral exophthalmia. Physician exam discovered an infected PNS evocating an infectious orbital complication, confirmed by radiography.

The treatment associated an emergency surgical drainage of the orbital complication, then local steroid. Ethmoidectomy was necessary in one case. During evolution, there was a stabilisation of the PNS and no sign of recidivism of the orbital infection.

\section{INTRODUCTION}

La polypose nasosinusienne (PNS) est une affection bénigne, rare chez l'enfant. Dans la majorité des cas, la symptomatologie inaugurale est dominée par l'obstruction nasale, la rhinorrhée et les troubles olfactifs.

Dans certains cas, l'exophtalmie peut être un motif de consultation de la PNS et signe une complication oculoorbitaire.

Nous présentons dans ce travail deux cas de PNS diagnostiqués suite à des complications oculo-orbitaires.

\section{OBSERVATION 1}

T. Mariem, âgée de 13 ans, sans antécédents pathologiques notables, a consulté au service d'ophtalmologie pour exophtalmie brutale gauche évoluant depuis une semaine, sans contexte infectieux ni traumatique.

L'examen ophtalmologique a objectivé une exophtalmie gauche non axile, des troubles de l'oculo-motricité avec une limitation des mouvements oculaires dans tous les sens associée à une baisse de l'acuité visuelle de l'œil gauche à $7 / 10$.
Devant ce tableau clinique, fait d'exophtalmie aiguë unilatérale chez une fille de 13 ans, une origine infectieuse ou tumorale a été suspectée.

A l'interrogatoire, la patiente a mentionné la notion d'obstruction nasale bilatérale, d'hyposmie évoluant depuis plusieurs mois et l'apparition de rhinorrhée purulente depuis une semaine non améliorée par l'Amoxicilline per os.

L'examen clinique des fosses nasales a montré la présence de sécrétions purulentes et des polypes comblant les deux fosses nasales : aspect de polypose nasosinusienne stade III des deux côtés.

Devant l'installation d'une exophtalmie aiguë chez une patiente présentant une PNS surinfectée, le diagnostic de rhinosinusite compliquée d'abcès orbitaire a été fortement évoqué. Une IRM du massif facial et orbitaire, faite en urgence, a montré un abcès sous périosté intra orbitaire gauche et un comblement ethmoïdal, des deux sinus maxillaires et des fosses nasales (figure 1). 


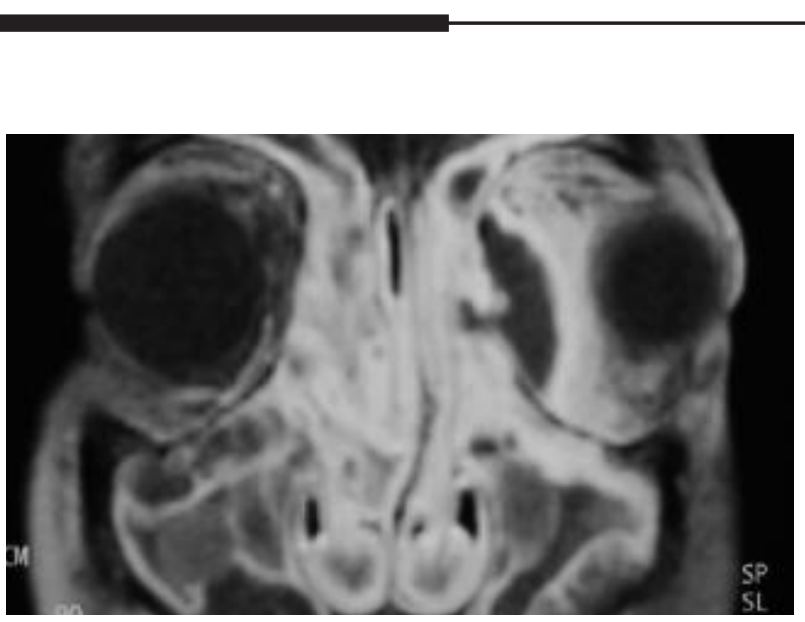

Fig. 1 : IRM du massif facial, coupe coronale T1 post-gadolinium: comblement pansinusien par un processus non Iytique, ne prenant pas le produit de contraste avec une image en hyposignal , biconvexe orbitaire interne évoquant un abcès sous périosté.

Un drainage chirurgical de l'abcès sous périosté a été réalisé en urgence par voie externe paracanthale interne avec mise d'une lame de drainage. Une antibiothérapie par voie parentérale et des lavages quotidiens au sérum bétadiné ont été institués (figure 2).

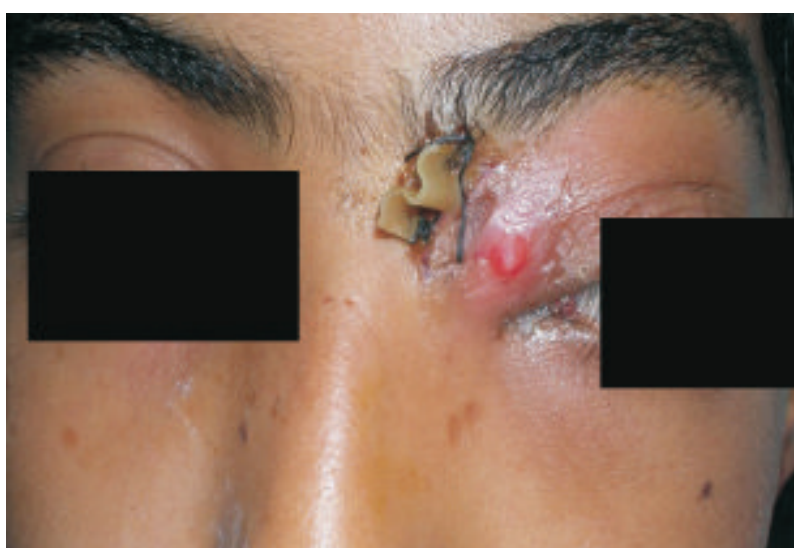

Fig. 2 : Incision de drainage paracanthale interne.

L'évolution a été marquée par une amélioration nette de l'état local, avec disparition de l'exophtalmie et une normalisation de l'acuité visuelle.

Un scanner du massif facial réalisé après deux semaines a montré la disparition de la collection orbitaire, avec un comblement partiel des cellules ethmoïdales et des sinus maxillaires (figure 3).

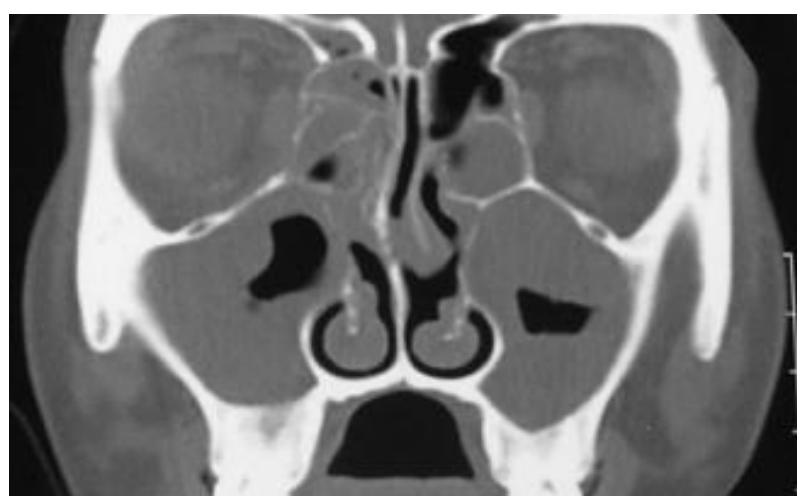

Fig. 3 : Scanner des sinus, coupe coronale, fenêtre parenchymateuse : comblement du sinus ethmoidal, épaississement en cadre de la muqueuse des sinus maxillaires et confinement méatal. Disparition de la collection intra orbitaire.

Un test à la sueur négatif a permis d'éliminer une mucoviscidose. La patiente a été mise sous corticothérapie locale et générale en cure courte. Contrôlé après 2 mois, l'examen clinique des fosses nasales a montré une régression nette des polypes. Nous avons décidé de poursuivre la corticothérapie locale. Le recul est de 12 mois.

\section{OBSERVATION 2}

$B$.Sofiane âgé de 13 ans sans antécédents pathologiques particuliers, a consulté pour un œdème palpébral supérieur gauche associé à une tuméfaction de l'angle interne de l'œil gauche évoluant depuis deux jours dans un contexte fébrile. L'interrogatoire a révélé la notion d'hyposmie, d'obstruction nasale bilatérale chronique et de ronflement non explorés.

L'examen de la face a trouvé une tuméfaction paracanthale gauche douloureuse et non fluctuante. L'examen ophtalmologique était au départ normal. L'examen endonasal a révélé un aspect de PNS stade IV surinfectée.

Le diagnostic de rhinosinusite compliquée de cellulite préseptale a été retenu. Le malade a été hospitalisé, mis sous antibiothérapie par voie générale. L'évolution a été marquée par l'apparition d'une exophtalmie gauche et d'une baisse de l'acuité visuelle. L' IRM d'urgence, orbitaire et du massif facial, a objectivé un abcès sous périosté gauche associé à une PNS stade IV. Le patient a bénéficié d'un drainage chirurgical de l'abcès sous périosté en urgence par voie canthale interne. L'évolution a été marquée par une nette amélioration de l'état local au troisième jour postopératoire. L'antibiothérapie a été maintenue pendant deux semaines et une TDM du massif facial de contrôle a montré la disparition de la collection orbitaire. Le patient a reçu une cure courte de corticoïdes par voie générale à la dose de $0,5 \mathrm{mg} / \mathrm{kg} / \mathrm{j}$ et a été mis sous corticothérapie locale. Cependant, l'évolution a été marquée par la récidive des surinfections et la persistance de la PNS. Une ethmoïdectomie bilatérale par voie endoscopique a été alors indi- 
quée. Les suites opératoires étaient simples. La corticothérapie locale a été reprise en post-opératoire immédiat. A 6 mois de recul l'examen était normal.

\section{DISCUSSION}

La PNS est une pathologie rare chez l'enfant. Wolf et Trighor (1) sur une période de 11 ans ont rapporté respectivement des séries de 124 et 102 enfants. Dans notre service nous avons colligé deux cas de PNS infantiles sur une période de 8 ans. La symptomatologie d'appel n'a rien de spécifique : il s'agit dans la plupart des cas d'une obstruction nasale chronique bilatérale, une rhinorrhée aqueuse, muqueuse voire mucopurulente, des troubles de l'odorat et des céphalées généralement fronto-orbitaires $(3,4)$.

Les complications infectieuses locorégionales de la PNS sont rares et constituent exceptionnellement un motif de consultation. Elles sont surtout intracrâniennes à type d'abcès frontal puis intra-orbitaires (5). En effet, $8 \%$ des infections orbitaires sont secondaires à une PNS (5).

Les facteurs qui favorisent ces complications sont : le traitement inadéquat ou non instauré des poussés des rhinosinusites, comme c'était le cas pour nos deux malades, la présence ou la coexistence d'un déficit immunitaire (diabète, aplasie médullaire, SIDA) et la présence d'anomalies acquises telle qu'une déhiscence ou une brèche méningée. Les complications oculo-orbitaires des rhinosinusites chroniques et de la PNS surviennent avec prédilection chez les enfants. Ceci est dû à la fragilité de l'os planum à cet âge et au drainage veineux intra-orbitaire des cellules ethmoïdales $(2,5)$.

Les stades d'infection orbitaire vont de la cellulite préseptale ou péri-orbitaire à l'abcès intraconique et la thrombophlébite du sinus caverneux. Ces différents stades de gravité croissante ne se succèdent pas forcement. En effet, le patient peut se présenter d'emblée à un stade avancé. Un de nos malades était diagnostiqué au stade d'abcès sous périosté, La gravité de ces complications réside dans le risque de cécité par atteinte du nerf optique et le pronostic vital peut être mis en jeu dans les formes atteignant l'apex orbitaire,la loge caverneuse et les structures cérébroméningées (7).

L'apport de l'imagerie dans le diagnostic positif de la PNS au stade de complications est indiscutable. En effet l'IRM est un examen sensible dans la mise en évidence et la stadification des complications orbitaires. Elle permet aussi de déceler une atteinte cérébrale associée à type d'abcès du cerveau ou de thrombophlébite. La TDM est plus spécifique dans la précision des lésions nasosinusiennes et leurs extensions locorégionales. Elle permet également de montrer d'éventuelles variantes anatomiques et de déhiscence osseuse soit de l'os planum, soit de la lame criblée de l'éthmoïde $(6,8)$

Le traitement des complications orbitaires dépend du stade de la complication. En cas d'abcès sous périosté, le traitement repose sur le drainage chirurgical associé à une antibiothérapie adaptée (9).

Le traitement de la PNS sous jacente est discuté selon les équipes. Certains auteurs préconisent un traitement médical reposant sur une corticothérapie locale et générale et une ethmoïdectomie en cas de récidive de la complication ou de non réponse au traitement médical de la PNS (10). D'autres auteurs sont partisans d'une ethmoïdectomie d'emblée afin de prévenir la récidive ou la survenue d'autres complications (11)

Pour notre première malade nous avons opté pour un traitement médical. Nous avons observé une amélioration nette de la symptomatologie clinique et régression de la PNS sans récidive de complication. Le recul est toutefois modeste (12 mois). Pour le deuxième malade une ethmoïdectomie d'emblée a été préconisée, vue la fréquence de la surinfection rhinosinusienne chez lui, mais aussi devant l'échec de la corticothérapie dans le traitement de la PNS.

\section{CONCLUSION}

Devant une exophtalmie d'installation aiguë chez un enfant, un examen ORL est indispensable pour chercher une étiologie nasosinusienne. La PNS, rarement à l'origine de cette complication, pose des controverses quant à sa prise en charge thérapeutique. L'âge de ces patients et la gravité de cette complication indiquent pour certains un traitement radical de la PNS.

\section{REFERENCES}

1-Bobin S., Monteil. J.ORL de l'enfant ; Médecine-Sciences Flammarion $1996: 104-108$.

2-.Aidan P, Bankova S. La polypose naso-sinusienne. Société Française d'ORL, Editeur 2000 :57-63.

3- Barry B., Ameline E. Complications orbitaires des sinusites de l'adulte. Ann Otolaryngol Chir Cervicofaciale $2000 ; 117$ (1) :19-25.

4- Peytral C., Chevalier E.. Complications ophtalmologiques en pathologie ORL; EMC -ORL. 2 OO4 ; $1: 199-224$.

5- Fadden $\mathrm{C}$. et al. Orbital complications of sinusitis in aspirin triad syndrome. The laryngoscope. $1996 ; 106$ ( 9) : 1103-7.

6 - Abgrall S., Broucker T. Abcès cérébral frontal, complication rare de la polypo- se nasosinusienne. Neurochirugie, 1995, 41 (5) : 367-71

7- Page $C$. et al. Abcès et empyèmes intracrâniens d'origine ORL. Ann Otolaryngo Chirugie Cervicofaciale $2005 ; 122$ (3):120-126.

8- Williams R.S, Carruth JAS ; Orbital infection secondary to sinusitis in children : diagnosis and mangement .OtolaryngoClin North Am 1992 ; 17: 550-7.

9- Kesser A, Berenholz. Transnasal endoscopic drainage of a medial subperiosteal orbital abscess. Eur Arch Otorhinolaryngol 1998;25:293-5.

10-Triglia JM, Belus JF: la polypose nososinusienne de l'enfant. Diognostic et problèmes thérapeutiques. Ann Pediatr (Paris) 1992; 39 (8) : 473-477.

11- Traglia JM, Dessi P.Intranasal ethmoidectomy in nasal polyposis in children. Indications and results . Int J Pediat Otorhinolaryngo. 1992; 23:125-131 